\title{
Shear stress-induced MMP1 and PDE2A expressions in coronary atherosclerosis
}

\author{
Ma YS ${ }^{1,2^{*}}$, Xie $\mathrm{YH}^{3^{*}}$, Ma D ${ }^{4}$, Zhang $\mathrm{JJ}^{4}$, Liu $\mathrm{HJ}^{1}$ \\ Department of Medical Imaging, Second Hospital of Hebei Medical University, Shijiazhuang, China. \\ huaijunliu1954@126.com
}

\section{ABSTRACT}

AIM: Biomechanical stress plays an essential role in coronary atherosclerosis (CAS), however, inter-relations between mechanical conditions and gene expressions remain unclear.

METHODS: We constructed finite element model of CAS to map human wall shear stress (WSS). Biopsy aortic tissue samples were obtained from 3 CAS patients. Gene expression pattern in CAS was analyzed by GEO datasets. Immunofluorescence staining and western blot confirmed protein expression and localization. RESULTS: Peak WSS was significantly increased in the vessel stenosis of CAS at $0.25 \mathrm{~s}$ (mean 55.1 $\mathrm{Pa}$ ). Analyses results of GSE76275 showed matrix metalloproteinases1 (MMP1) and phosphodiesterase2A (PDE2A) up-regulation in endothelial shear responsiveness, which was further validated and localized in vascular endothelial cells, smooth muscle cells and other cells by double immunofluorescence staining. Western blotting assay demonstrated up-regulation of MMP1 and PDE2A expression dependent on the WSS. CONCLUSIONS: MMP1 and PDE2A up-regulations rely on increased WSS in development and risk of CAS, suggesting that their elevation may be potential target for diagnosis and treatment (Fig. 3, Ref. 28). Text in PDF www.elis.sk

KEY WORDS: atherosclerosis, finite element analysis, gene expression, biomechanical stress.

\section{Introduction}

Coronary atherosclerosis (CAS), characterized by pathologic alteration of the atherosclerotic plaque, such as thinning of the fibrous cap and the development of a large necrotic core, predisposes the plaque to rupture, which then triggers thrombosis luminal, is a leading cause of cardiovascular diseases (CVD) (1-3). In combination with mechanical loading exerted by blood pressure, progressive atherosclerosis takes place(4). And then plaque rupture occurs when the stress developed in the vessel exceeds the yield strength of the material. Despite advanced understanding of the biology of CAS, however, in the mutual interactions between biomechanical conditions and underlying molecular mechanism leading to

\footnotetext{
${ }^{1}$ Department of Medical Imaging, Second Hospital of Hebei Medical University, Shijiazhuang, China, ${ }^{2}$ Department of Radiology, Shijiazhuang Hospital of Traditional Chinese Medicine, Shijiazhuang,China, ${ }^{3}$ Department of Pharmacy, Hebei University of Science and Technology, Shijiazhuang, China, and ${ }^{4}$ School of Public Health, North China University of Science and Technology, Tangshan, China

"Yan-shan Ma and Ying-hua Xie contributed equally to this work.

Address for correspondence: H.J. Liu, Department of Medical Imaging, Second Hospital of Hebei Medical University, Shijiazhuang, China.

Acknowledgments: This work was supported by Natural Science Foundation of Hebei Province (No. H2016106072) and Scientific and Technical Research Foundation of the Higher Education Institutions of Hebei Province, China (No. ZD2017032).
}

aggressive stenosis and ultimately occlusion, rupture of unstable plaques and formation of thrombosis are so far poorly understood.

Gene expression profiling have been widely used for understanding molecular mechanisms of CAS, whether mechanical quantities such as wall stresses and strains, referred to as "mechanotransduction," are associated with gene expression is little known. Generally, vascular endothelial cells (ECs) first sense hemodynamic changes, and then express a unique transcriptional profile under very high wall shear stress known to induce expansive arterial remodeling (5). Studies have reported that hemodynamic mechanical forces may be sufficient to alter the EC and inflammatory regulation of the growth characteristics of VSMCs $(6,7)$, however, details regarding the gene expression patterns in vascular tissue-specific cells associated with mechanotransduction in CAS context remain unknown.

With recent advances in patient-specific finite element analysis (FEA), now allowing an accurate computation of these mechanical quantities in the various cardiovascular diseases $(8-10)$, the investigation of the inter-relation between gene expression and mechanical properties of the CAS is possible. A simplified biomechanical and clinical study has indicated that wall stress is probably a better predictor of rupture risk, and recently it has been shown that FEA can give a prediction of stress values in individual aneurysms using geometry derived from CT scan data (11).

Hence, we hypothesized that the formation of CAS creates a specific mechanical environment, which activates molecular programs for development and instability of plaques at the CAS pro- 
cess. To test this hypothesis, we employed a range of clinical case samples and gene expression detection as well as FEA model to investigate the interaction between local gene expression patterns and biomechanical conditions contributing to aggressive stenosis effects in a population of CAS, which is crucial for the prevention of CVD.

\section{Materials and methods}

Human arteries harvest

Three human coronary artery tissue samples were obtained from organ donors. Each coronary aorta was carefully and thoroughly examined for the presence or absence of atherosclerosis by microscopic examinations of consecutively dissected serial segments of the entire artery by cardiovascular pathologists and a segment of the proximal atherosclerosis for normal aorta as a control. Arteries were cleaned of adjacent adipose and myocardial tissue in explanted hearts (17). The adventitial layer remained intact. Each specimen was averagely divided into two parts, one was embedded in paraffin and the other was snap-frozen in liquid nitrogen and stored at $-80^{\circ} \mathrm{C}$.

\section{Finite element analysis (FEA)}

FEA is a computational modeling technique used for detailed CAS morphology, hemodynamic forces, and mechanical properties to determine the mechanical stress distribution as described previously in detail (12). Finite element analyses were performed using ANSYS Workbench 14.0 (ASN Systems Ltd, Cannonsburg, USA) to determine patterns and values of wall shear stress.

\section{Microarray data information acquisition}

The mRNA expression profiling of GSE132651 was based on Human Genome Gene Chip arrays GPL96 and included 13 samples (endothelial cells from living subjects with early coronary atherosclerosis) and 6 subjects with normal coronary endothelial function. (Submission date: Jun 01, 2020) (13).

\section{Immunofluorescence staining}

Immunofluorescence staining was performed as described previously (14). The primary antibodies were mouse anti-MMP1 (1:50, 36665, R\&D Systems) and rabbit anti-PDE2A (1:100, MA525395 , Invitrogen). Images were captured by confocal microscopy (DM6000 CFS, Leica) and processed by LAS AF software. All images of primary cells were taken with $63 \times$ NA 0.75 objectives.
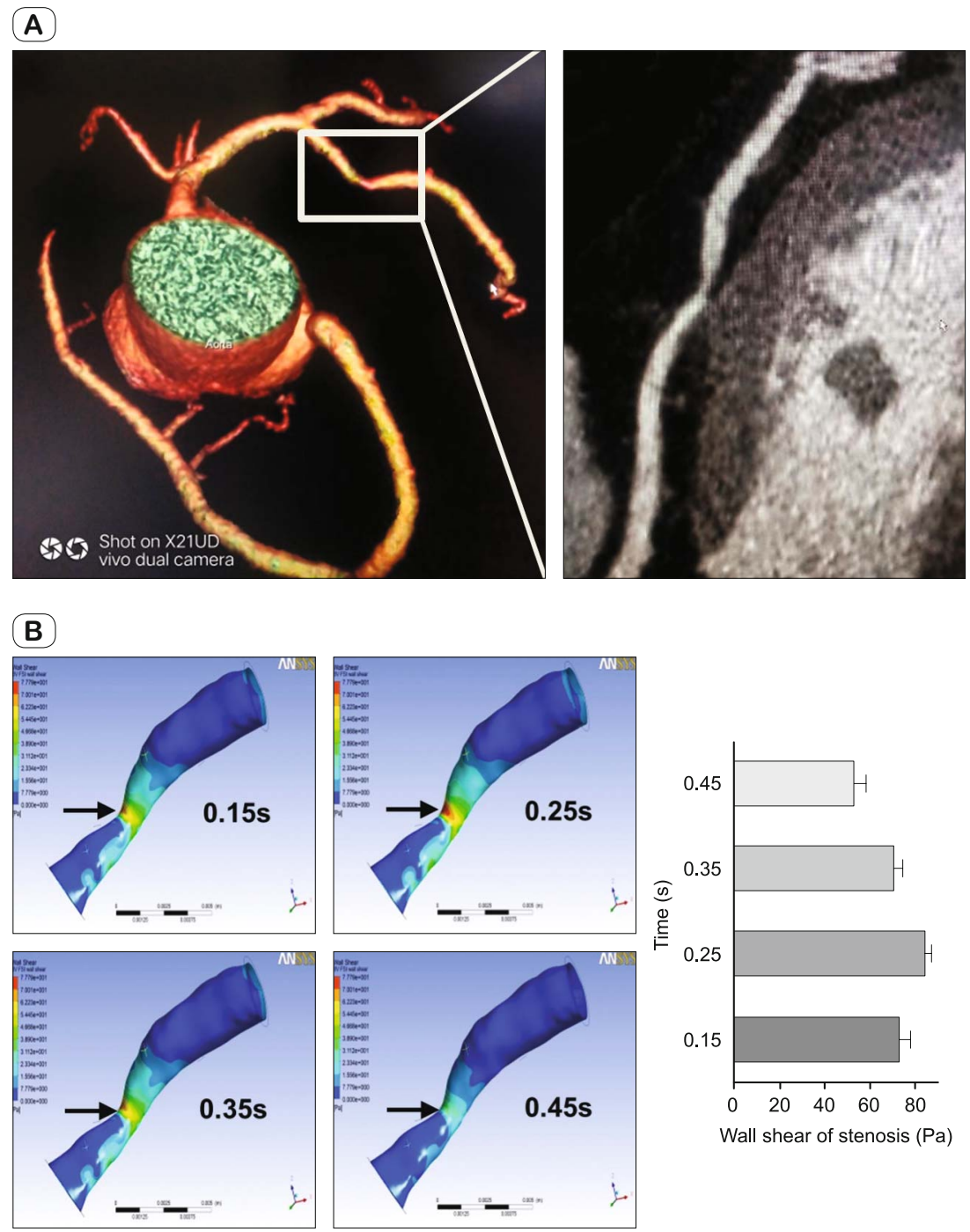

Fig.1. Results of CT scanning and finite element analysis (FEA) in patients with CAS. A) Representative photographs of $\mathrm{CT}$ scans of participating patientswere converted into $3 \mathrm{D}$ reconstructions. B) By finite element (FEM) analysis, a three- dimensional wall shear stress (WSS) distribution results of coronary artery stenosis at different calculating time points. Red color corresponds to highest wall shear stress whilst blue for lowest wall stress. 
(A)

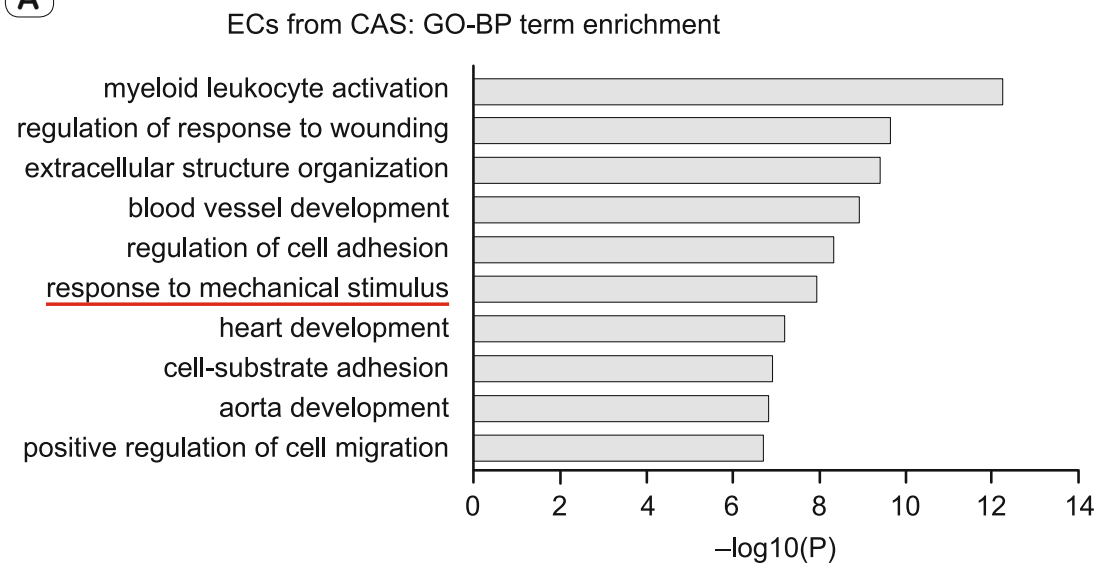

(B)

\begin{tabular}{|c|c|}
\hline Fold & \\
\hline 1.48 & MMP1 \\
\hline 1.35 & PIEZO2 \\
\hline 1.15 & SERPINE2 \\
\hline 0.82 & CXCR4 \\
\hline 0.21 & JUP \\
\hline 1.37 & TGFBR2 \\
\hline 1.21 & PSPH \\
\hline 2.13 & PDE2A \\
\hline 1.61 & COL3A1 \\
\hline 1.14 & CYBA \\
\hline 1.02 & STAT1 \\
\hline 0.20 & CRADD \\
\hline-0.08 & USP53 \\
\hline 0.41 & PIEZO1 \\
\hline-0.22 & PKDREJ \\
\hline 0.31 & COL1A1 \\
\hline
\end{tabular}

(C)
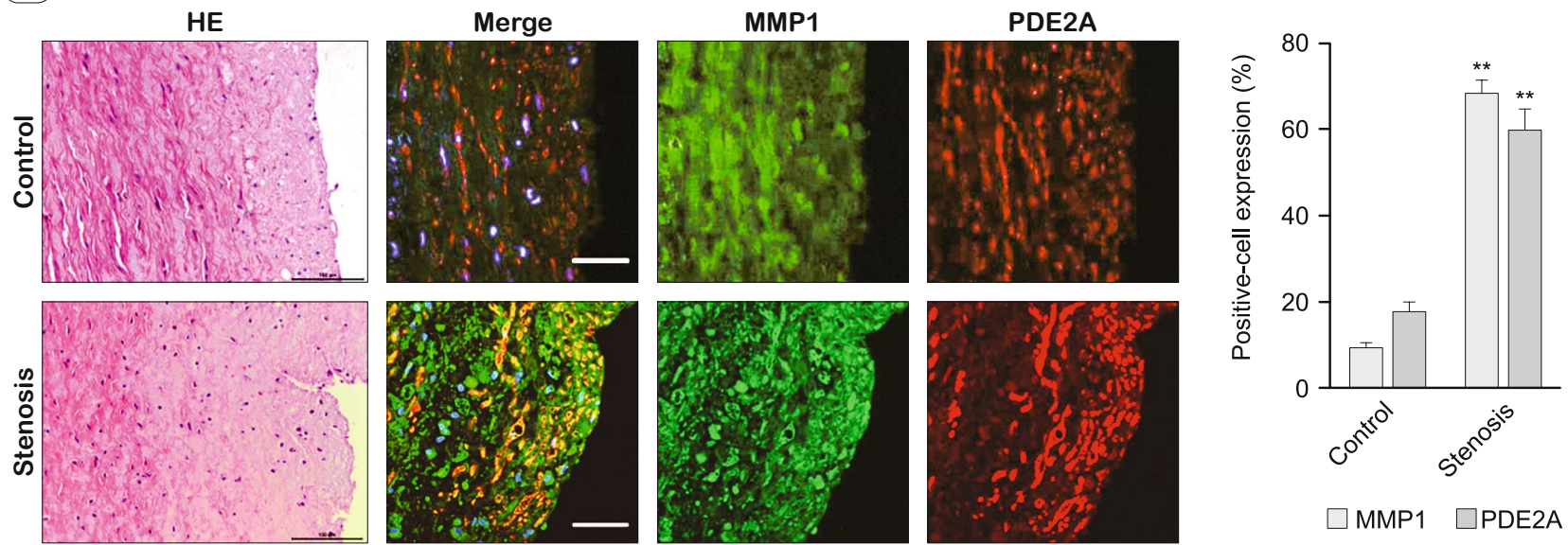

$\square$ MMP1 $\square$ PDE2A

Fig. 2. MMP1 and PDE2A expression is up-regulated in the human CAS tissues. A) The GO analysis for enrichment of the differentially expressed genes in endothelial cells from 13 living subjects with early CAS and 6 subjects with normal coronary endothelial function (Control) based on the biological process. B) Hierarchical clustering of the response to mechanical stimulus-associated different expressed genes in CAS and in control. C) Double immunofluorescence staining of MMP1, PDE2A, and DAPI was performed. Scale bars $=50 \mu \mathrm{m}$. Right: statistical analysis of MMP1 and PDE2A positive cells in human CAS and control tissues. $\mathbf{n}=\mathbf{5}$ for each group. $* * \mathbf{p}<0.01$ vs Control.

Spearman's correlation. Two-sided $\mathrm{p}<0.05$ were considered significant and denoted with 1,2 , or 3 asterisks when lower than 0.05 , 0.01 , or 0.001 , respectively.

\section{Results}

Wall shear stress (WSS) alteration at different parts of CAS

Patient-specific WSS computation by FEA model was performed in 9 patients with severe CAS according to computed tomography scans with corresponding 3-dimensional volume-rendered images (Fig. 1A).In constructed FEA model, the WSS distribution maps of CAS at $0.15,0.25,0.35$, and $0.45 \mathrm{~s}$ were calculated and obtained. Among them, the WSS alteration was the most obvious at $0.25 \mathrm{~s}$ for vascular stenosis region (Fig.1B).
Identification of higher levels of MMP1 and PDE2A expression in human CAS tissues

Because atherosclerosis preferentially occurs at specific sites of the vasculature where endothelial cells (ECs) are exposed to disturbed blood flow (15), differential expression genes of endothelial cells from 13 living subjects with early coronary atherosclerosis and 6 subjects with normal coronary endothelial function of GSE132651 were obtained.Gene ontology (GO) analysis revealed that enriched genes in human aortic ECs were strongly associated with response to mechanical stimulus (Fig. 2A), including 16 differential expression genes (Fig. 2B).Among detected genes, the most up-regulated MMP1 (4.48-fold) and PDE2A (2.13-fold) genes were further verified in human CAS tissues $(n=3)$ than that in normal coronary tissues $(n=3)$ by double immunofluorescence staining analysis (MMP1: 67.4\% vs 9.8\%, p <0.01; PDE2A: 59.6\% 


\section{A}

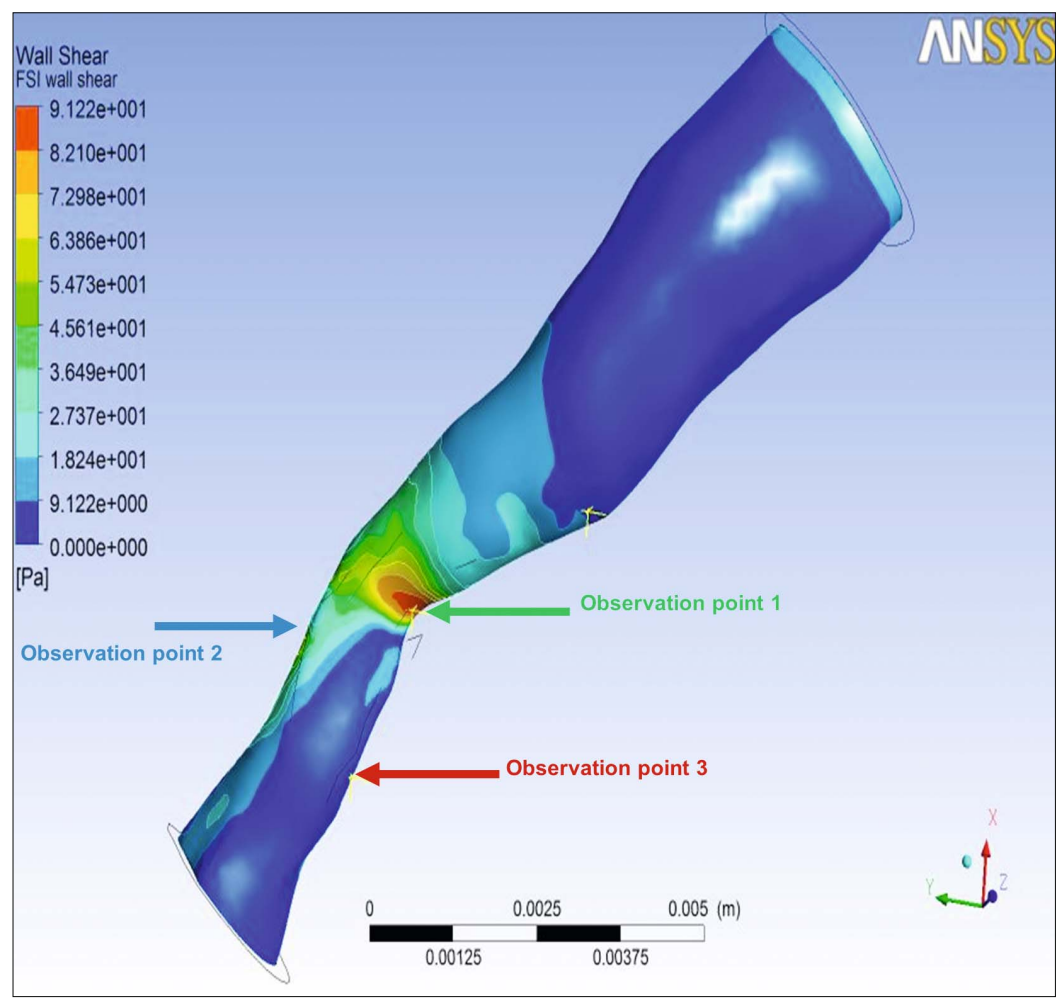

\section{B}

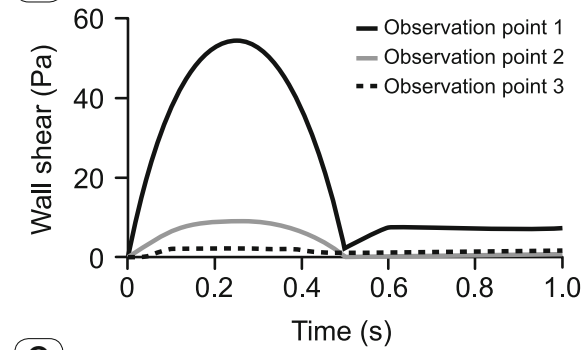

(C)
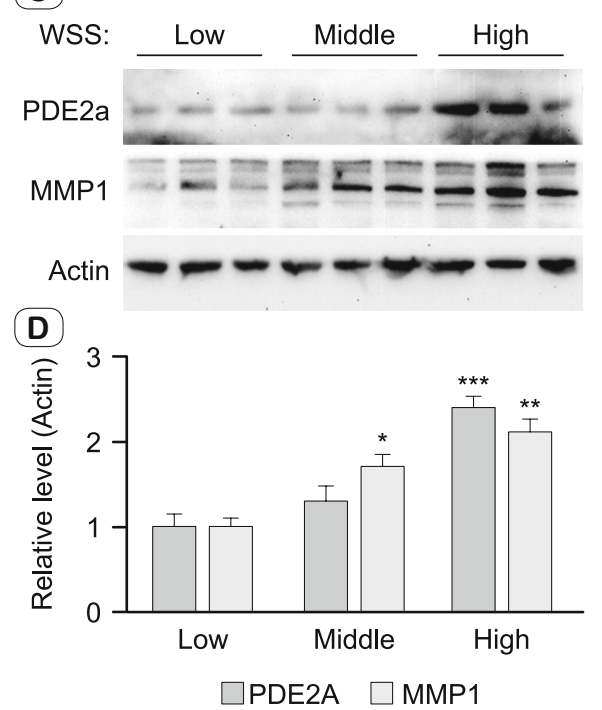

Fig. 3. WSS-dependent effects on MMP1 and PDE2A expression in human CAS tissues. A) FEA provides a three -dimensional WSS distribution of the CAS. Arrows in different colors point to corresponding regions of WSS alteration. Green arrow indicates high stress, blue arrow indicates middle stress and red arrow indicates low stress. B) Curves of stress measurements in different observation points. C) Western blotting analysis of the protein levels of MMP1 and PDE2A. D) MMP1 and PDE2A protein abundance is differentially induced in human CAS tissues from areas of low, middle and high WSS. $\mathrm{n}=3$ for each group. ${ }^{*} \mathrm{p}<0.05, * * \mathrm{p}<0.01$ and $* * * \mathrm{p}<0.001$ vs Low group.

vs $18.7 \%, \mathrm{p}<0.01)$. In addition, we also found that MMP1 and PDE2A were not only localized with vascular ECs, but also localized with other cell types (such as infiltrating inflammatory cells and vascular smooth muscle cells (Fig. 2C).

MMP1 and PDE2A are significantly increased dependent on WSS

To clarify whether the up-regulations of MMP1 and PDE2A expression at CAS is an effect of WSS alteration, we set different observation points in FEA model of CAS, which were before stenosis (position 1), stenosis (position 2) and after stenosis (position 3) (Fig. 3A). As shown in Figure 3B, the maximumvalue of WSS $(54.3 \pm 1.7 \mathrm{~Pa})$ occurred at stenosis (position 1). Furthermore, western blotting assay of MMP1 and PDE2A protein expressions at low, middle and high region of WSS in corresponding CAS tissues represent association with WSS in dose-dependent manner (Fig. $3 \mathrm{C}$ ), and statistical analysis quantifies these alterations. Compared to low WSS region, MMP1 and PDE2A relative expression in middle WSS region are $1.56(\mathrm{p}<0.05)$ and $1.34(\mathrm{p}>0.05)$ respectively, MMP1 and PDE2A relative expression in high WSS region are $2.11(p<0.01)$ and $2.47(p<0.001)$ respectively (Fig. 3D).

\section{Discussion}

This study investigated inter-relation between FEA-determined WSS and the local gene expression of corresponding tissue areas from patients with CAS. Significantly, coronary artery stenosis regions with a high postulated WSS showed an increased expression of MMP1 and PDE2A genesinvolved in degrading ECM components in comparison withlow-WSS areas. Differences in biomechanical regionwere associated with MMP1 and PDE2A expressions patterns, suggestingthat the heterogeneous gene expression could be due to different states of CAS progression.

MMP1 is extracellular proteolytic enzyme that degrades various components of the extracellular matrix (ECM) and induces vascular remodeling, it has been implicated in the disruption of atherosclerotic plaques that leads to acute coronary events(16). Although up-regulation of MMP1 expression responding to vascular mechanical stimulus was firstly found in ECs from early CAS patients (13), which is also proved in vitro,mediating fluid shear stress induced angiogenic sprouting (17), it simultaneously drives vascular smooth muscle cell de-differentiation and resteno- 
sis (18). In this study, we also found its expression in multi-type cells in human CAS tissues, indicating that it may be an important therapeutic target for CAS. As originally reported, inhibition of MMP1 expression in ECs (19) or VSMCs (20) attenuates vascular remodeling. And the latest research also shows that glucagon-like peptide 1 treatment reverses vascular remodeling by down-regulating MMP1 expression (21).

PDE2A as a member of the cyclic nucleotide phosphodiesterase family (PDE),controls the levels of the second messenger cAMP and cGMP in many cell types including endothelial cells. It is mainly expressed in brain tissue (22), adrenal glands and vascular endothelial cells, and it is related to vascular permeability and neutrophil extravasation $(23,24)$. Existing research shows that PDE2 inhibition augments cGMP and cAMP signaling to elicit pulmonary dilation and prevent pulmonary vascular remodeling (25). In addition, pan-cGMP PDE inhibitor zaprinast administration enhances muscle microvascular blood flow and improves the vascular and metabolic actions of insulin in skeletal muscle. These researches imply that targeting specific cGMP PDE2A may provide a promising avenue for development of a novel class of therapeutics for cardiovascular diseases. Although the relationship between MMP1 and PDE2A expression in cardiovascular diseases has not yet been reported, MMP1 acts as a downstream target of NO- cGMP signal pathway contributing to the pathological pulmonary vascular remodeling in PH (26). Furthermore, recombinant adenoviruses mediated-overexpression of endothelial nitric oxide synthase (eNOS) treatment in rabbit showed a decreasing tendency in regions with MMP1 staining and regressed atherosclerosis (27). Therefore, it is reasonable to speculate that the PDE2A-MMP1 signaling axis may play a key regulating role in biomechanical stress-induced formation and progress of coronary atherosclerosis.

There are three limitations to the current implementation of our FEA technique. First, FEA model is only static linear elastic to characterize the vessel WSS magnitude and distribution in CAS, so time-varying factors related to dynamic features of the applied loading (e.g. strain rate, load frequency) may be neglected. The latest results in model verification are promising. Secondly, the influence of the vascular tunica adventitia tissues, including connective and adipose tissue was neglected in our FEA model. We expect exclusion of these tissues to have a minimal effect on the peak load-induced stress environment in the coronary artery system. Last, in order to establish relationship between mechanical conditions and the local expression of gene in CAS, a larger study population has tobe analyzed in a multicenter approach. However, in this study, aninteresting relation between local calculated CAS WSS from FEA and regional MMP1 and PDE2A gene expression was found for the first time, that might play a potentially relevant role in the maintenance of plaque stability and repression of CAS progress.

Gene expression results, especially degradation of the ECM components, were in accord with histological observations based on preoperative biomechanical wall stress calculations (28). According to previous research reports and our confirmation, MMP1 and PDE2A expression could be potential targets for diagnosis and treatment of CAS. The pathophysiology of mechanotransduction has to be examined in further molecular and biomechanical studies.

\section{References}

1. Roth GA, Forouzanfar MH, Moran AE et al. Demographic and epidemiologic drivers of global cardiovascular mortality. N Engl J Med 2015; 372 (14): 1333-1341.

2. Lippi G, Franchini M, Targher G. Arterial thrombus formation in cardiovascular disease. Nat Rev Cardiol 2011; 8 (9): 502-512.

3. Davies MJ. Stability and instability: two faces of coronary atherosclerosis. The Paul Dudley White Lecture 1995. Circulation 1996; 94 (8): 2013-2020.

4. Jiang Y, Peng W, Teng $\mathbf{Z}$ et al. Local blood pressure associates with the degree of luminal stenosis in patients with atherosclerotic disease in the middle cerebral artery. Biomed Eng Online 2016; 15 (1): 67.

5. Dolan JM, Sim FJ, Meng $\mathbf{H}$ et al.Endothelial cells express a unique transcriptional profile under very high wall shear stress known to induce expansive arterial remodeling. Am J Physiol Cell Physiol2012; 302 (8): $1109-1118$.

6. Russo TA, Stoll D, Nader HB et al. Mechanical stretch implications for vascular endothelial cells: Altered extracellular matrix synthesis and remodeling in pathological conditions. Life Sci 2018; 213: 214-225.

7. Qi YX, Han Y, Jiang ZL. Mechanobiology and Vascular Remodeling: From Membrane to Nucleus. Adv Exp Med Biol 2018; 1097: 69-82.

8. Liu S, Wang F, Liu R. Fluid flow and fluid shear stress in canaliculi induced by external mechanical loading and blood pressure oscillation. Appl Math Mech 2015; 36 (005): 681-692.

9. Erhart P, Schiele $\mathbf{S}$, Ginsbach $\mathbf{P}$ et al.Gene Expression Profiling in Abdominal Aortic Aneurysms After Finite Element Rupture Risk Assessment. J EndovascTher 2017; 24 (6): 861-869.

10. Koseki H, Miyata H, Shimo S et al. Two Diverse Hemodynamic Forces, a Mechanical Stretch and a High Wall Shear Stress, Determine Intracranial Aneurysm Formation. Transl Stroke Res 2019; 11 (1): 80-92.

11. Kurt F, Phillip S, Gautam M et al. Alterations in phenotype and gene expression of adult human aneurysmal smooth muscle cells by exogenous nitric oxide. Exp Cell Res. 2019, 384 (1): 111589.

12. Martin D, Boyle F. Finite element analysis of balloon-expandable coronary stent deployment: Influence of angioplasty balloon configuration. Int J Numer Method Biomed Eng 2013; 29 (11): 1161-1175.

13. Hebbel RP, Wei P, Milbauer $L$ et al.Abnormal endothelial gene expression associated with early coronary atherosclerosis. J Am Heart Assoc 2020; 9 (14): e016134.

14. Ma D, Chang LY, Zhao S et al. KLF5 promotes cervical cancer proliferation, migration and invasion in a manner partly dependent on TNFRSF11a expression. Rep, 2017, 7 (1): 15683.

15. Zhang $\mathbf{C}$, Zhou $\mathbf{T}$, Chen $\mathbf{Z}$ et al.Coupling of Integrin $\alpha 5$ to Annexin A2 by Flow Drives Endothelial Activation. Circ Res 2020.

16. Marina B M, Amélie V, Galina K S et al. Redundancy of IL-1 Isoform Signaling and Its Implications for Arterial Remodeling. PLoS ONE, 2016; 113 (3): e0152474.

17. Galie PA, Nguyen DH, Choi CK et al. Fluid shear stress threshold regulates angiogenic sprouting. Proc Natl Acad Sci USA 2014; 111 (22): 7968-7973. 


\section{7-292}

18. Austin KM, Nguyen N, Javid G et al.Noncanonical matrix metalloprotease-1-protease-activated receptor-1 signaling triggers vascular smooth muscle cell dedifferentiation and arterial stenosis. J Biol Chem2013; 288 (32): 23105-23115.

19. Game BA, Xu M, Lopes-Virella MF et al. Regulation of MMP-1 expression in vascular endothelial cells by insulin sensitizing thiazolidinediones. Atherosclerosis 2003; 169 (2): 235-243.

20. Bryan AG, Alejandro M, Lin $\mathbf{H}$ et al.Pioglitazone inhibits MMP-1 expression in vascular smooth muscle cells through a mitogen-activated protein kinase-independent mechanism. Atherosclerosis 2005; 178 (2): 249-256.

21. Fan SH, Xiong QF, Wang $L$ et al. Glucagon-like peptide 1 treatment reverses vascular remodelling by downregulating matrix metalloproteinase 1 expression through inhibition of the ERK1/2/NF- $\mathrm{kBsignalling}$ pathway. Mol Cell Endocrinol 2020: 111005.

22. Salpietro V, Perez-Dueñas B, Nakashima K et al.A homozygouslossof-functionmutation inPDE2Aassociated to early-onset hereditary chorea. Mov Disord 2018; 33 (3): 482-488.

23. Naganawa M, Waterhouse RN, Nabulsi $\mathbf{N}$ et al. First-in-human assessment of the novel PDE2A PET radiotracer 18F-PF-05270430.J Nucl Med2016; 57 (9): 1388-1395.
24. Chen W, Spitzl A, Mathes D et al. Endothelial Actions of ANP Enhance Myocardial Inflammatory Infiltration in the Early Phase After Acute Infarction. Circ Res 2016, 1192 (2): 237-248.

25. Bubb KJ, Trinder SL, Baliga RS et al. Inhibition of phosphodiesterase 2 augments cGMP and cAMP signaling to ameliorate pulmonary hypertension. Circulation 2014; 1306 (6): 496-507.

26. Zou L, Xu X, Zhai Z et al. Identification of downstream target genes regulated by the nitric oxide-soluble guanylate cyclase-cyclic guanosine monophosphate signal pathway in pulmonary hypertension. J Int Med Res 2016; 443 (3): 508-519.

27. Hayashi T, Sumi D, Juliet PA et al.Gene transfer of endothelial NO synthase, but not eNOS plus inducible NOS, regressed atherosclerosis in rabbits. Cardiovasc Res 2004; 612 (2): 339-351.

28. Erhart P, Grond-Ginsbach C, Hakimi M et al.Finite element analysis of abdominal aortic aneurysms: predicted rupture risk correlates with aortic wall histology in individual patients. J EndovascTher2014; 21 (4): $556-564$.

Received September 5, 2020. Accepted October 1, 2020. 